\title{
CNFs@CNTs: Superior Carbon for Electrochemical Energy Storage**
}

\author{
Jian Zhang ${ }^{+}$Y Yong-Sheng Hu*, Jean-Philippe Tessonnier ${ }^{+}$, Gisela Weinberg ${ }^{+}$, Joachim Maier*, \\ Robert Schlögl ${ }^{+}$, and Dang Sheng $\mathrm{Su}^{+}$
}

\author{
* Max-Planck-Institut für Festkörperforschung, 70569 Stuttgart (Germany) \\ E-mail: y.hu@fkf.mpg.de; s.weiglein@fkf.mpg.de \\ ${ }^{+}$Department of Inorganic Chemistry, Fritz-Haber-Institut der Max-Planck-Gesellschaft, Faradayweg 4-6, \\ 14195, Berlin (Germany) \\ E-mail: dangsheng@fhi-berlin.mpg.de
}

** The authors are indebted to the Max Planck Society and acknowledge support within the framework of the ENERCHEM project. The authors would like to acknowledge technical assistance from the Combicat Centre, Universiti Malaya (Kuala Lumpur, Malaysia); Edith Kitzelmann, Fritz-Haber-Institut; and Dr. Andreja Gajović, Molecular Physics Laboratory, Rudjer Boskovic Institute (Zagred, Croatia). Supporting Information is available online from Wiley InterScience or from the author.

Carbon nanotubes are hollow cylinders of pure carbonand the nanochannels within them are big enough toaccommodate various atoms and even small molecules.Recently published research showed that carbon nanopeapods (e.g., $\mathrm{C}_{60} @$ SWNTs_-single-wall nanotubes filled with fullerenes) exhibit unusual electronic and mechanical properties when compared to empty nanotubes.[1] Selective incorporation of foreign carbons into CNTs could indeed improve the spatial occupancy inside the CNT channels in a controlled manner, thereby providing a potential way to optimize their porosities for various applications such as gas adsorption, heavy metal ion removal, and energy storage. However, while carbon hybrids with chemical continuity in the nanostructure are extremely interesting from a fundamental scientific point of view, wider practical applications thereof are yet to be found.

Lithium-ion rechargeable batteries are one of the greatest successes in modern electrochemistry as is evident in their broad application in consumer electronics and more importantly green energy storage as well as their potential use in hybrid electric vehicles.[2] As a readily available and low-cost resource, carbon is a promising negative electrode material for lithium secondary batteries. However, along with the commercialization of Li-ion batteries, limits in performance have been reached for currently available synthetic/natural graphite electrode materials. Next-generation electrodes are expected to have a higher reversible capacity as well as a superior cycling stability. The rapid development in the field of carbon nanofilaments, especially since the microscopic observation of single walled nanotubes (SWNTs), provides us with a great opportunity to achieve these goals.[3] Nonetheless, there is still a number of problems to be overcome prior to any large-scale application of these filaments. Firstly, it is an enormous challenge to achieve long-term stability at the same time as high reversible capacity. CNTs with a high surface area usually exhibit a high reversible capacity of $400-600 \mathrm{~mA} \cdot \mathrm{h} \cdot \mathrm{g}^{-1}$, but show weak stability even after various surface modifications.[4] Secondly, portable lithium batteries are strictly limited in size, but commercial CNT products always possess a loose macroscopic structure, hence low bulk density. Novel CNT based electrodes with a spatially more compact structure are therefore expected to provide better storage capacities.

Here we report, for the first time, the template-free synthesis of carbon nanotube encapsulated carbon nanofibers (CNFs@CNTs), where cheap and low-quality commercial CNTs are transformed into highperformance electrode materials. Compared to SWNTs, the CNTs used in this study had a lower surface area $\left(82 \mathrm{~m}^{2} \cdot \mathrm{g}^{-1}\right)$, bigger outer diameter $(50-200 \mathrm{~nm})$, and thicker walls (50-100 walls). Large-scale production reduced their price to as little as 50 USD per kilogram. The CNFs@CNTs produced with our method exhibited a reversible capacity of $410 \mathrm{~mA} \cdot \mathrm{h} \cdot \mathrm{g}^{-1}$ during 120 charge/discharge cycles when used as the anode in lithium based batteries. Taking into account the low price of the feedstock, the straightforward process, and the high performance of CNFs@CNTs, our route is extremely promising for future applications. Our study opens a new pathway for the synthesis and application of novel nanocarbons in all fields of materials science and technology. 
As illustrated in Scheme 1, CNFs@CNTs were synthesized via selective deposition of an active metal on the inner walls of CNTs (step 1), which was followed by the growth of CNFs by means of catalytic chemical vapor deposition (CCVD, step 2). The Co@CNTs precursor, containing 0.5 wt\% Co, was produced using a capillary force based incipient wetness impregnation methods, whereby a thin layer of Co nitrate solution is preferentially dispersed onto the inner surface of the CNTs. This causes the generation of metallic Co nanoparticles (average size: $6.6 \mathrm{~nm}$ ) primarily on the inner wall of the CNTs (Fig. 1) during the subsequently performed $\mathrm{H}_{2}$ reduction step. These then act as active phase for CNF growth in the tubular chamber during the CCVD process.

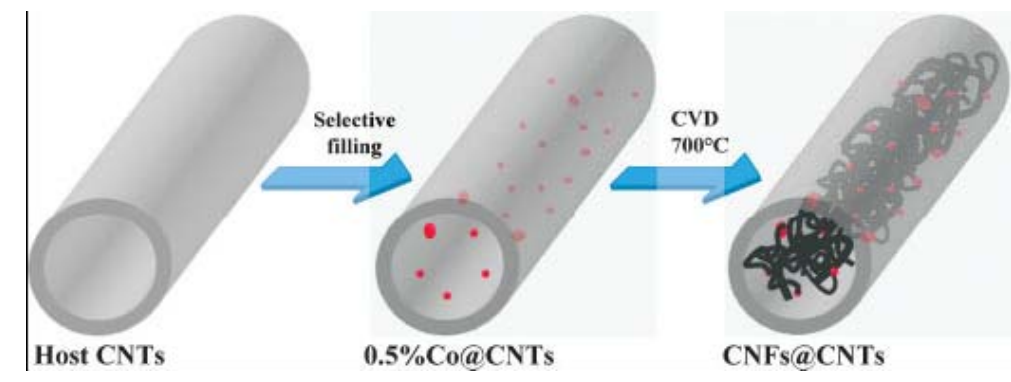

Scheme 1. Synthesis route to carbon-nanotube-encapsulated carbon nanofibers (CNFs@CNTs).

Figure 2 compares the morphologies of pristine, commercially available CNTs and as-synthesized CNFs@CNTs. Commercial CNTs exhibit an inner diameter of 20-80 nm and a generally open characteristic, which allows both, the solution Co precursor and the hydrocarbon gas, to diffuse easily into the tubular channel. No obvious difference in the external appearance of pristine CNTs and CNFs@CNTs was identified by scanning electron microscopy (SEM), indicating good confinement of assynthesized CNFs inside the encapsulating CNTs. High-resolution SEM images of some open ended CNFs@CNTs revealed that the tube channels were filled with small CNFs (see insert Fig. 2b). As can be seen in the transmission electron microscopy (TEM) images shown in Figures $2 \mathrm{c}$ and $2 \mathrm{~d}$, as-synthesized CNFs are of an open characteristic, approx. $10 \mathrm{~nm}$ in outer diameter and $0.2 \mu \mathrm{m}$ in length. Tilting the TEM specimen to $\pm 30^{\circ}$ (Figs. 2e-f) confirmed the good confinement of CNFs, which mainly benefits from the successful preferential deposition of active metal nanoparticles onto the inner walls. Most nanotubes with open ends were full of CNFs while there was a small number of bamboo structured/ completely closed nanotubes which were hollow due to the absence of initial Co nanoparticles.

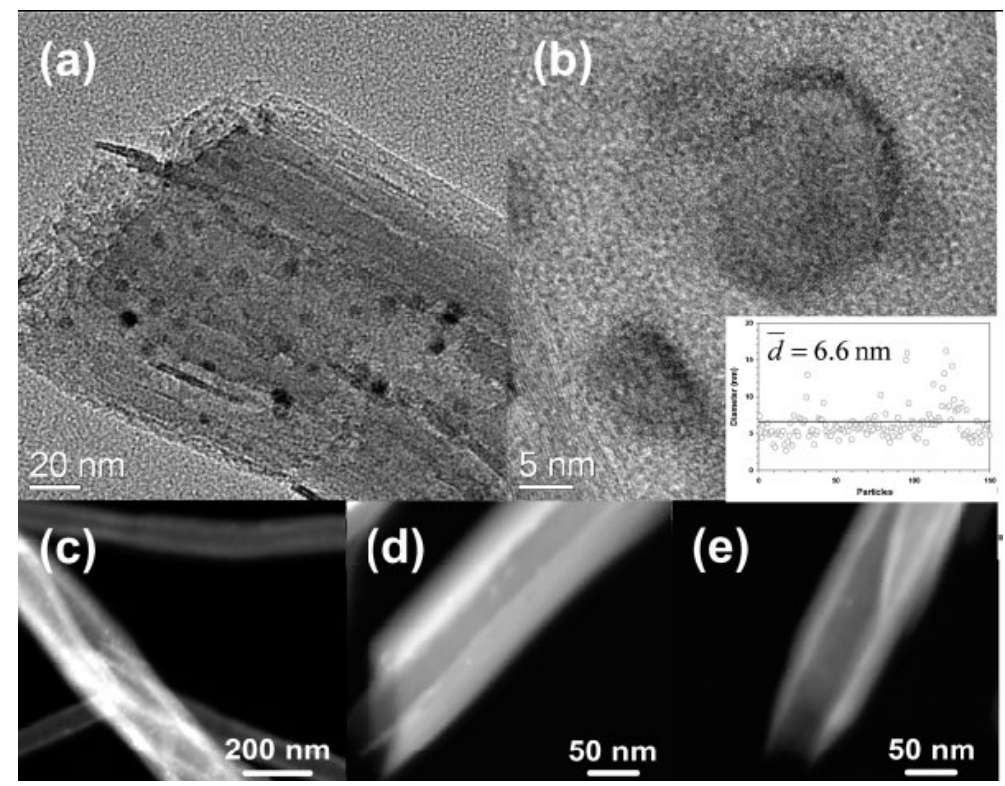

Figure 1. Transmission electron microscopy (TEM) images of $0.5 \% \mathrm{Co} @ \mathrm{CNTs}$ after reduction with $\mathrm{H}_{2}$. a,b) Bright-field high-resolution (HR)TEM images. c-e) Dark-field scanning TEM images.

The CNFs@CNTs with a novel structure possessed a much better porosity than the pristine CNTs. $\mathrm{N}_{2}$ physisorption tests showed that the specific surface area and the pore volume increased from $82 \mathrm{~m}^{2} \cdot \mathrm{g}^{-1}$ 
and $0.17 \mathrm{~cm}^{3} \cdot \mathrm{g}^{-1}$ to $347 \mathrm{~m}^{2} \cdot \mathrm{g}^{-1}$ and $0.61 \mathrm{~cm}^{3} \cdot \mathrm{g}^{-1}$, respectively (Fig. 3a). The total increase in weight was $25 \%$ as measured after the CCVD process; which suggests a greatly improved utilization of space inside the hollow channels of the CNTs, thus an increase in their bulk density. This arises primarily from the formation of secondary pores between the CNFs and CNTs as well as the extremely close stacking of CNFs inside the CNTs, because the produced CNFs alone could not contribute to such a great extent.[5] As was revealed in Raman experiments (Fig. 3c), fresh CNTs with $0.5 \mathrm{wt} \%$ Co showed $D$ and $G$ bands at 1354 and $1579 \mathrm{~cm}^{-1}$, respectively, which are close to the theoretical values for graphite. Such a highly ordered structure arises from the fact that commercial CNTs are treated at approx. $2700{ }^{\circ} \mathrm{C}$ during manufacturing. After the CCVD process, the position of the $G$ and $D^{\prime}$ bands is almost unchanged while the $D$ band shifted downwards from 1354 to $1330 \mathrm{~cm}^{-1}$. The slight increase in the ratio of $D$ and $G$ band intensities, $\mathrm{R}=\mathrm{I}_{(\mathrm{D})} / \mathrm{I}_{(\mathrm{G})}$, from 0.5 to 1.1 revealed that there is still a high degree of graphitization but some disordered carbon is also present in the CNFs@CNTs. At this point it is worth mentioning that the templated synthesis of carbon tube-in-tube materials (CTITs) recently became a hot issue in materials science.[6] However, compared to CTITs ourCNFs@CNTs exhibit better spatial utilization of the hollow channels present in CNTs, and thus a higher porosity with more potential application in many important fields, e.g., hydrogen storage and waste water treatment for environmental protection.

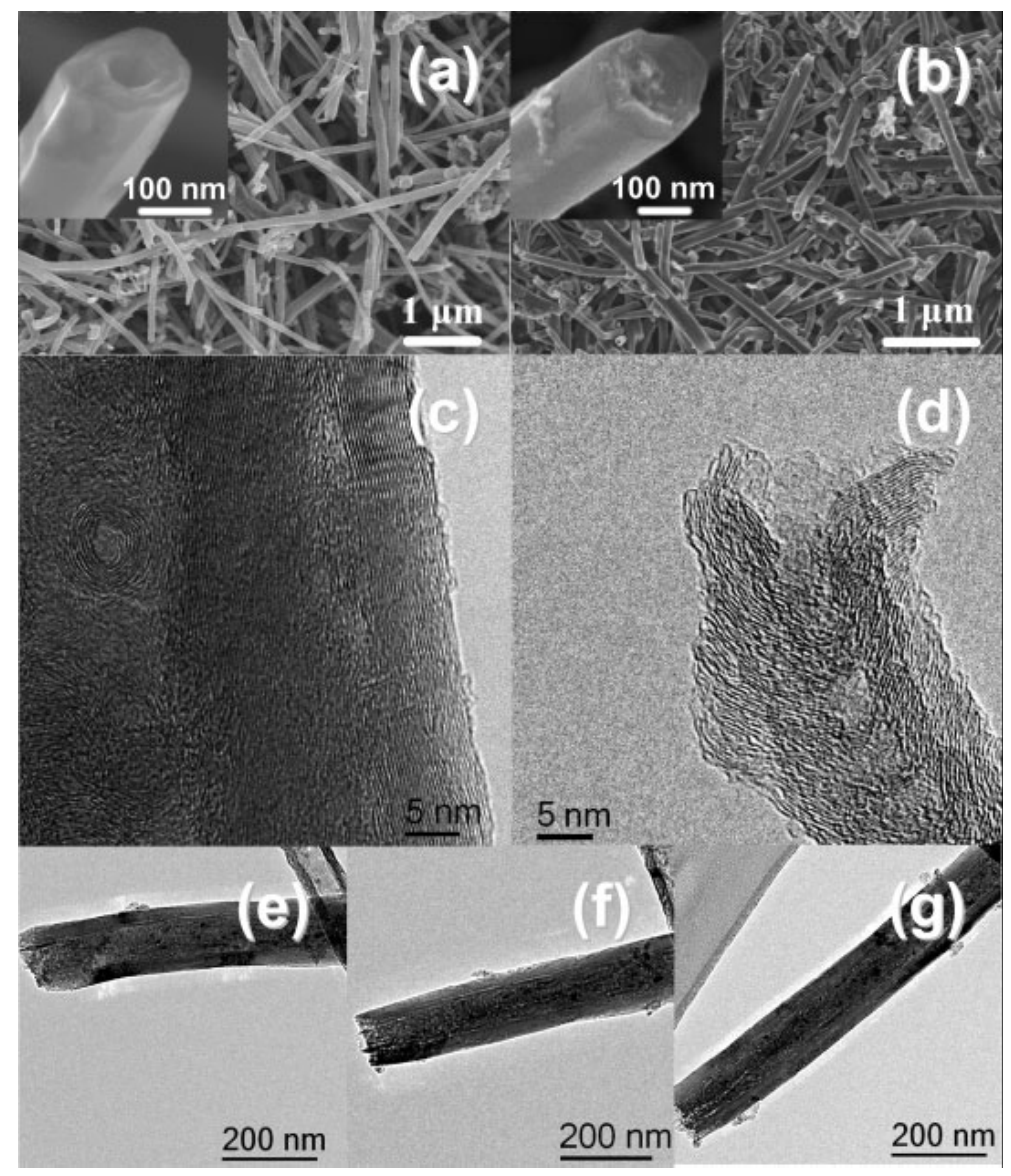

Figure 2. Morphologies of pristine CNTs and as-prepared CNFs@CNTs. a) Scanning electron microscopy (SEM) image of fresh CNTs. b) SEM image of CNFs@CNTs. c) HRTEM image of CNFs@CNTs. d) HRTEM image of typically synthesized CNFs. eg) TEM images of CNFs@CNTs with the sample holder tilted to $-30^{\circ}, 0^{\circ}$, and $30^{\circ}$, respectively.

In the course of the last decade, carbon nanofilaments attracted great attention as electrode materials in electrochemical devices. Therefore, we investigated the performance of CNFs@CNTs in preliminary Li insertion/extraction experiments. Figure 4a shows the discharge/charge ( $\mathrm{Li}$ insertion/ extraction) curves of a CNFs@CNTs electrode cycled in a 1m solution of $\mathrm{LiPF}_{6}$ in 1:1 (v/v) ethylene carbonate (EC)/ dimethyl carbonate (DMC) as electrolyte at a rate of $\mathrm{C} / 5$ (one lithium per six formula units $\left(\mathrm{LiC}_{6}\right)$ in five hours). In both, discharge and charge curves, extended flat plateaus can be observed. Additionally, reduction and oxidation peaks corresponding to $\mathrm{Li}$ intercalation and deintercalation into the grapheme layers can be identified in the cyclic voltammogram curve (Fig. 4b). The sloped regions in the discharge/charge curves can be attributed to Li insertion/extraction into the disordered structure of the CNFs@CNTs electrode. A large irreversible capacity in the first discharge and charge process is most likely due to the formation of a 
solid electrolyte interphase (SEI), which is backed up by the disappearance of the reduction peak at around $0.6 \mathrm{~V}$ in the second cycle.[7,8] Unlike ethylene carbonate, propylene carbonate (PC) is considered as a safe low-temperature electrolyte.[8,9] However, the PC solvent and the solvated $\mathrm{Li}^{+}$ions tend to cointercalate into graphite accompanied by severe exfoliation of graphite layers and thus destruction of the graphite structure.[8,9] What is very noteworthy is the excellent cycling performance in a PCbased electrolyte at a rate of $\mathrm{C} / 5$ (Fig. 4c). Nevertheless, $\mathrm{PC}$ and solvated $\mathrm{Li}^{+}$ions tend to co-intercalate into the graphite accompanied by severe exfoliation of graphite layers and thus destruction of the graphite structure. $[8,9]$.
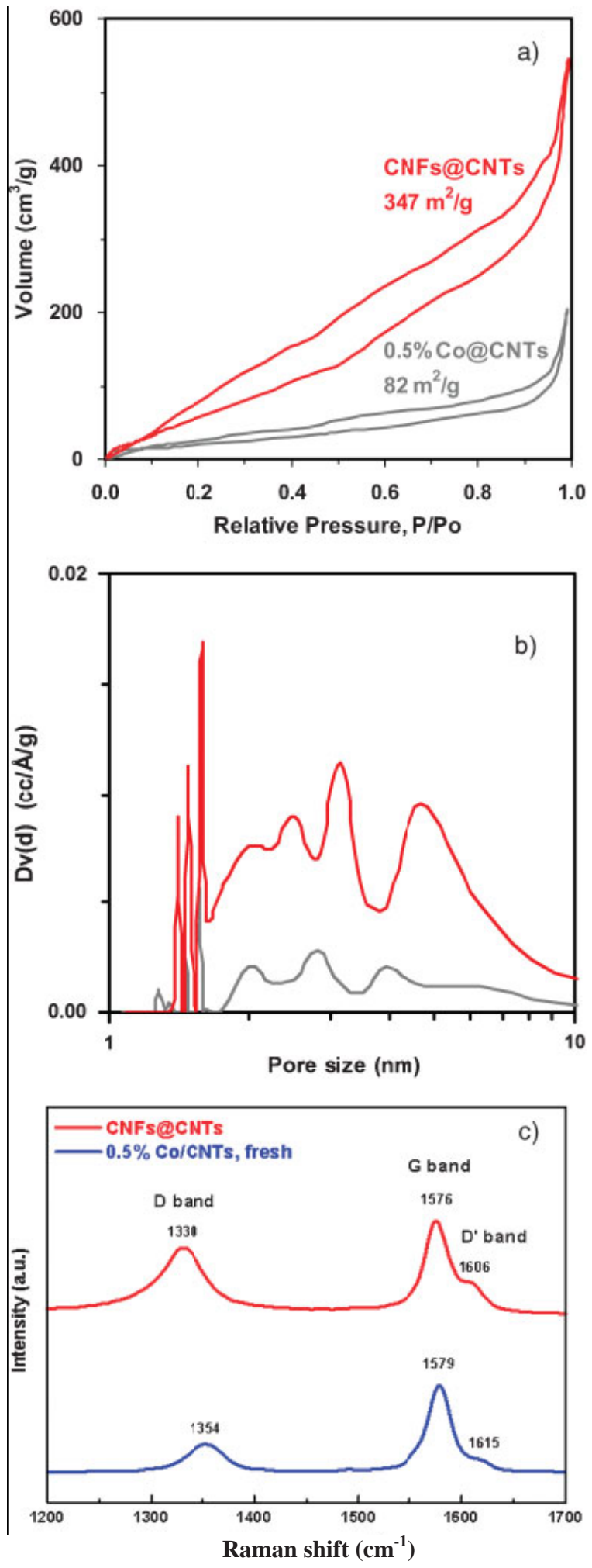

Figure 3. Characterization results of 0.5\%Co@CNTs and CNFs@CNTs samples. a) $\mathrm{N}_{2}$ adsorption isotherms. b) Pore size distributions. c) Raman spectra. 
In terms of permanence of the high lithium storage capacity, CNFs@CNTs are superior to pristine CNTs. During 120 cycles the reversible capacity of the CNFs@CNTs electrode stayed at around $410 \mathrm{~mA} \cdot \mathrm{h} \cdot \mathrm{g}^{-1}$ while it gradually decreased to $258 \mathrm{~mA} \cdot \mathrm{h} \cdot \mathrm{g}^{-1}$ when the electrode was formed from commercial CNTs (Fig. 4d). Furthermore, the volumetric ratio of $\mathrm{C}_{\mathrm{rev}, \mathrm{CNFs} @ \mathrm{CNTs}}: \mathrm{C}_{\mathrm{rev}, \mathrm{CNTs}}$ reached a value as high as 2.02, with the 25\% increase in bulk density being already accounted for.[10] CNFs@CNTs also possessed a high rate capability. When the discharge/charge rate was increased from $\mathrm{C} / 5$ to $1 \mathrm{C}$, the reversible capacity remained higher than $300 \mathrm{~mA} \cdot \mathrm{h} \cdot \mathrm{g}^{-1}$ during 50 cycles (data not shown). CNFs@CNTs electrodes show a much higher reversible capacity and rate capability compared to synthetic/natural graphite anodes in commercial Li-ion batteries. The superior stability of CNFs@CNTs probably arises from a steric hindrance effect of their compact structure which suppresses the diffusion of big electrolyte molecules through wall defects. Their outstanding cycling performance in combination with their high storage capacity makes CNFs@CNTs much more attractive than other carbon materials previously reported in the literature, e.g., MWNTs, hard carbon, and CNFs.
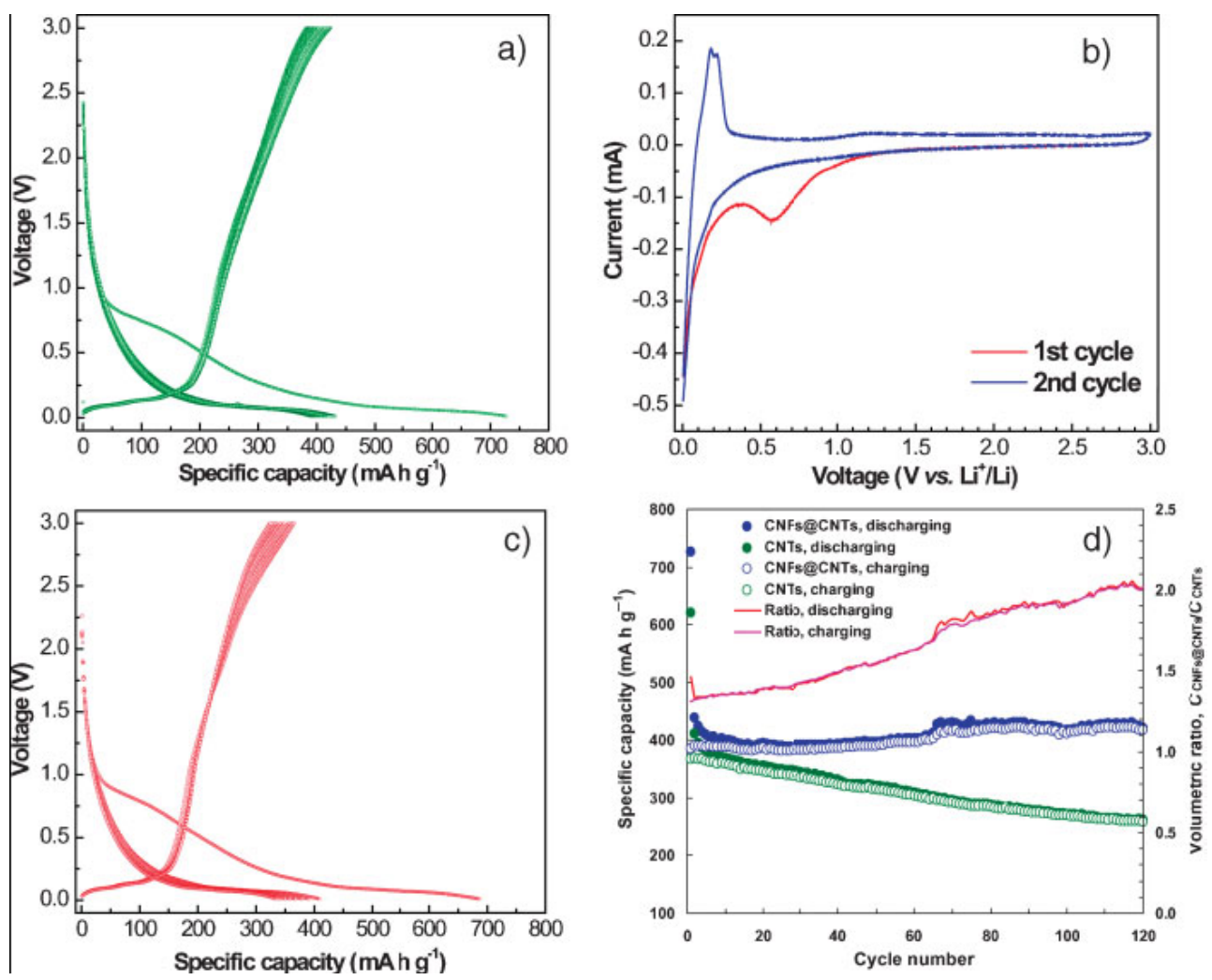

Figure 4. Electrochemical performance of CNFs@CNTs. a) Galvanostatic discharge/charge (Li insertion/extraction, voltage decrease/increase) curves of CNFs@CNTs at a cycling rate of $\mathrm{C} / 5$ in $1 \mathrm{M} \mathrm{LiPF} 6$ in 1:1 (v/v) ethylene carbonate (EC)/dimethyl carbonate (DMC). b) Cyclic voltammogram of CNFs@CNTs at a scan rate of $0.1 \mathrm{mV} \cdot \mathrm{s}^{-1}$ in the range of $0.01-3 \mathrm{~V}$ in $1 \mathrm{M} \mathrm{LiPF} 6$ in EC/DMC solution. c) Galvanostatic discharge/charge curves at a cycling rate of $\mathrm{C} / 5$ in $1 \mathrm{M} \mathrm{LiClO}_{4}$ in propylene carbonate (PC) solution. d) Comparison of the electrochemical performance of pristine CNTs and CNFs@CNTs in 1m LiPF 6 in EC/ DMC solution.

In summary, our study provides a simple route for the transformation of cheap commercial CNTs into highly efficient carbon for electrochemical energy storage applications. For this synthesis route to prepare CNFs@CNTs the feedstock is cheap and each operation is well-established and readily industrialized. Due to a higher spatial utilization CNFs@CNTs displayed a reversible volumetric capacity which is twice as high as that of pristine CNTs as well as an outstanding long-time stability. Their unique structural properties make CNFs@CNTs a new class of carbon materials with significant potential for applications in the field of gas adsorption, environmental protection, fuel cells, catalysis, hydrogen storage, etc. Our method can be extended to: (i) other carbon materials (mesoporous carbon, activated carbon, carbon nanocones, etc.) and (ii) one-dimensional inorganic nanotubes/ nanofibers ( $\mathrm{BN}_{\mathrm{X}}, \mathrm{GaAs}, \mathrm{SiO}_{2}, \mathrm{ZnO}$, etc.).

\section{Experimental}

Commercial carbon nanotubes (PR-24-HHT, Applied Sciences, Inc.) were refluxed in concentrated nitric acid at $130{ }^{\circ} \mathrm{C}$ for $10 \mathrm{~h}$ and then dried at $100{ }^{\circ} \mathrm{C}$ overnight. Cobalt $(0.5 \mathrm{wt} \%)$ was deposited inside the 
functionalized CNTs via a modified incipient wetness impregnation method, whereby - driven by capillary forces - a solution of cobalt nitrate in water/ethanol (90:10) was filled into the tube channels of the host CNTs. The obtained material was then dried at $100{ }^{\circ} \mathrm{C}$ for $10 \mathrm{~h}$ and calcined at $350{ }^{\circ} \mathrm{C}$ for $2 \mathrm{~h}$ in air, before being reduced at $400{ }^{\circ} \mathrm{C}$ under $\mathrm{H}_{2}$ flow. The $0.5 \% \mathrm{Co} @ \mathrm{CNFs}$ catalyst was then heated to 700 ${ }^{\circ} \mathrm{C}$ in a $1: 2 \mathrm{H}_{2} / \mathrm{He}$ mixture $\left(30 \mathrm{ml} \cdot \mathrm{min}^{-1}\right)$ after which a 1:1:2 mixture of $\mathrm{C}_{2} \mathrm{H}_{4}: \mathrm{H}_{2}: \mathrm{He}\left(40 \mathrm{ml} \cdot \mathrm{min}^{-1}\right)$ was introduced to the catalyst bed. The chemical vapor deposition (CVD) process was maintained for 20 min after which the temperature was cooled down to ambient temperature in He flow.

Lithium intercalation/deintercalation tests were carried out in two-electrode Swagelok-type cells. The working electrodes were prepared by mixing each carbon sample $(90: 10 \mathrm{w} / \mathrm{w})$ with poly (vinyl difluoride) (PVDF) and pasting the mixture on pure $\mathrm{Cu}$ foil (99.6\%, Goodfellow). Glass fiber (GF/D, Whatman) and pure lithium foils (Aldrich) served as separator and counter electrode, respectively.

The electrolyte consisted of a $1 \mathrm{M} \mathrm{LiPF}_{6}$ solution in ethylene carbonate (EC)/dimethyl carbonate (DMC) $(1: 1 \mathrm{v} / \mathrm{v})$ obtained from Ube Industries $\mathrm{Ltd}$ or a $1 \mathrm{M} \mathrm{LiClO}_{4}$ solution in propylene carbonate (PC). The cells were assembled in an argon-filled glove box. Electrochemical performance was tested for different current densities in the voltage range of $0.01-3 \mathrm{~V}$ using an Arbin MSTAT battery test system. Cyclic voltammetry measurements were performed with a VoltaLab 80 electrochemical workstation at a scan rate of $0.1 \mathrm{mV} \cdot \mathrm{s}^{-1}$. Nitrogen sorption isotherms and textural properties were determined at $-196{ }^{\circ} \mathrm{C}$ on a Quantachrome Autosorb automated gas sorption system. Surface areas were calculated using the BET method and total pore volumes were determined from the amount of nitrogen adsorbed at $p / p_{0} \approx 0.99$. Micro-Raman spectra were recorded on a Jobin Yvon LabRam spectrometer using a $632.8 \mathrm{~nm}$ excitation laser line. SEM images were recorded using a Hitachi S4800 scanning electron microscope.TEMand STEM images were recorded on Philips CM200 FEG and CM200 LaB $_{6}$ transmission electron microscopes operating at $200 \mathrm{kV}$. XRD and Raman spectra were recorded on a Philips PW3710 X'PERT diffractometer with $\mathrm{Cu} \mathrm{K}_{\alpha}$ radiation and a Dilor Z-24 spectrometer with a coherent Innova-100 Ar ion laser $(\lambda=514.5 \mathrm{~nm})$ as excitation source, respectively.

[1] See, e.g., a) B. W. Smith, M. Monthioux, D. E. Luzzi, Nature 1998, 396, 323. b) J. Sloan, A. I. Kirkland, J. L.Hutchison,M. L.H.Green, Chem. Comm. 2002, 1319. c) I. V. Krive, R. I. Shekhter, M. Jonson, Low Temp. Phys. 2006, 32, 887. d) J. W. Kang, H. J. Hwang, Nanotechnology 2004, 15, 1825.

[2] See, e.g., a) A.-M. Cao, J.-S. Hu, H.-P. Liang, L.-J. Wan, Angew. Chem. Int. Ed. 2005, 44, 4391. b) J. Maier, Nat. Mater. 2005, 4, 805. c) M. Endo, C. Kim, K. Nishimura, T. Fujino, K. Miyashita, Carbon 2000, $38,183$.

[3] See, e.g., a) A. Oberlin, M. Endo, T. Koyama, J. Cryst. Growth 1976, 32, 335. b) S. Iijima, Nature 1991, 354, 56. c) Y. Zhang, T. Ichihashi, E. Landree, F. Nihey, S. Iijima, Science 1999, 285, 1719.

[4] See, e.g., a) G. T. Wu, C. S. Wang, X. B. Zhang, H. S. Yang, Z. F. Qi, P. M. He,W. Z. Li, J. Electrochem. Soc. 1999, 146, 1696. b) S. H. Ng, J. Wang, Z. P. Guo, J. Chen, G. X. Wang, H. K. Liu, Electrochim. Acta 2005, 51, 23. c) R. S. Morris, B. G. Dixon, T. Gennett, R. Raffaelle, M. J. Heben, J. Power Sources 2004, 138, 277. d) Y. A. Kim, M. Kojima, H. Muramatsu, S. Umemoto, T. Watanabe, K. Yoshida, K. Sato, T. Ikeda, T. Hayashi, M. Endo, M. Terrones, M. S. Dresselhaus, Small 2006, 2, 667. e) J. M. Rosolen, E.Y.Matsubara, M. S. Marchesin, S. M. Lala, L. A. Montoro, S. Tronto, J. Power Sources 2006, 162, 620. f) M.-S. Park, S. A. Needham, G.-X. Wang, Y.-M. Kang, J.-S. Park, S.-X. Dou, H.-K. Liu, Chem. Mater. 2007, 19, 2406.

[5] If the increase in surface area, SA, was mainly due to the formation of CNFs, the surface area of CNFs@CNTs could be represented by $0.25 \cdot \mathrm{SA}_{\mathrm{CNFs}}+0.75 \cdot \mathrm{SA}_{\mathrm{CNTs}}=347 \mathrm{~m}^{2} \cdot \mathrm{g}^{-1}$, with $\mathrm{SA}_{\mathrm{CNTs}}=82 \mathrm{~m}^{2} \cdot \mathrm{g}^{-1} \cdot$ In which case SA0CNFs would equal $1142 \mathrm{~m}^{2} \cdot \mathrm{g}^{-1}-$ an impossibly high value for CNFs which are $10 \mathrm{~nm}$ in diameter, whose surface areas are typically reported to be lower than $500 \mathrm{~m}^{2} \cdot \mathrm{g}^{-1}$.

[6] See, e.g., a) G. L. Che, B. B. Lakshmi, E. R. Fisher, C. R. Martin, Nature 1998, 393, 346. b) Z. P. Zhu, D. S. Su, G. Weinberg, R. E. Jentoft, Robert. Schlögl, Small 2005, 1, 107. c) F. S. Ou, M. M. Shaijumon, L. J. Ci, P. M. Ajayan, Carbon, 2007, 45, 1713.

[7] J. R. Dahn, T. Zheng, Y. Liu, J. S. Xue, Science 1995, 270, 590.

[8] M. Winter, O. J. Besenhard, M. E. Spahr, P. Novak, Adv. Mater. 1998, 10, 725.

[9] G. H. Wrodnigg, T. M. Wrodnigg, J. O. Besenhard, M. Winter, Electrochem. Commun. 1999, 1, 148.

[10] The volumetric ratio of the reversible capacities $\left(\mathrm{mA} \cdot \mathrm{h} \cdot \mathrm{cm}^{-3}\right)$ was calculated after normalizing the reversible capacities $\left(\mathrm{C}_{\mathrm{rev}}, \mathrm{mA} \cdot \mathrm{h} \cdot \mathrm{g}^{-1}\right)$ to the bulk density $\left(\rho, \mathrm{g} \cdot \mathrm{cm}^{-3}\right)$, i.e, $\mathrm{C}_{\mathrm{rev}, \mathrm{A} \rho \mathrm{A}} / \mathrm{C}_{\mathrm{rev}, \mathrm{B} \rho \mathrm{B}}$, with $\rho_{\mathrm{CNFs} @ \mathrm{CNTs}}=1.25 \cdot \rho_{\mathrm{CNTs}}$. 